\title{
DIOSCÓRIDES RESCATADO POR LOS ÁRABES
}

\author{
Ricardo H. Elía \\ Centro Islámico de la República. Buenos Aires \\ Argentina
}

Resumen: La historia oficial afirma que Occidente heredó directamente el legado cultural de Grecia y Roma. Pero si la mayoría de los manuscritos griegos y latinos fueron destruidos a partir del saqueo de la Biblioteca de Alejandría en 391, los archivos de Roma fueron devastados en sendas ocasiones entre 410 y 476 por visigodos y hérulos, y los escasos vestigios clásicos que quedaban en Atenas fueron arrasados por Justiniano I en 529, ¿cuál fue la conexión que logró transmitir esa literatura y pudo ser aprovechada en un período anterior al renacimiento? La salvación de las piezas literarias se realizó durante ochocientos años de activa y responsable tarea de recopilación por parte de los árabes durante la línea de tiempo que oscila entre 650-1450 en la que fueron rescatados, traducidos y retransmitidos hacia los cuatro puntos cardinales. Los científicos árabes, al igual que Arquímedes o Herón, nunca se separaron del saber empírico, por el contrario, lo profundizaron. El movimiento científico árabe no sólo interpretó el saber de los antiguos sino desarrolló una nueva ciencia donde la razón sustentaba a la fe y viceversa. Esta fue la base esencial que permitió construir el Renacimiento y la Ilustración, que a su vez posibilitaron la Modernidad. En este contexto, fue de capital importancia la traducción al árabe de la obra de Dioscórides ya que no sólo fue de gran utilidad para la farmacología y medicina en el mundo musulmán sino que a través de éste las recetas del médico griego de Nerón reingresaron a la Europa latina donde fueron recibidas como una panacea.

Palabras claves: Farmacología, veneno, hierbas medicinales, botánica, droga.

Abstract: Official history affirms that the West directly inherited the cultural legacy of Greece and Rome. But if most of the Greek and latin manuscripts they were destroyed at the sacking of the Library of Alexandria in 391, the archives of Rome were devastated in several occasions between 410 and 476 by Visigoth and heruls, and the little classic vestiges that were in Athens were devastated by Justinian in 529, which was the connection that managed to transmit that Literature and could be taken advantage of it in a period previous to the Renaissance? The salvation of the literary mastepieces was made during eight hundred years od active and responsible task of compilation by the Arabs during the time line that oscillates between 650-1450 in that were rescued, translated and relayed towards the four cardinal points. The Arab scientists, like Archimedes or Hero, never separated of the empirical knowledge, on the contrary, they deepened it. The Arab scientific movement not only interpreted the 
knowledge of the old ones but it developed the new science where the reason sustained the faith and vice versa. This was the essential base that allowed to construct to the Renaissance and the Illustration, that made possible Modernity as well. In this context, it was of capital importance the translation to the Arab of the work of Dioscorides since it was not only very useful for the pharmacology and medicine in the Muslim World but that through this one the recipes of the Greek physician of Nero turned back to latin Europe where were received like a panacea.

Key words: Pharmacology, poison, medical herbs, botany, drug.

Correspondencia: Ricardo H. Elía. Director del Departamento de Estudios Históricos del Centro Islámico de la República Argentina. Av. San Juan 3053 (1233) Buenos Aires, Argentina. Tel. (54-11) 4931-3577 (interno 113). ricardohselia@yahoo.com.ar

La historia oficial afirma que Occidente heredó directamente el legado cultural de Grecia y Roma. Pero si la mayoría de los manuscritos griegos y latinos fueron destruidos a partir del saqueo de la Biblioteca de Alejandría en 391, los archivos de Roma fueron devastados en sendas ocasiones entre 410 y 476 por visigodos y hérulos, y los escasos vestigios clásicos que quedaban en Atenas fueron arrasados por Justiniano I en 529, ¿cuál fue la conexión que logró transmitir esa literatura y pudo ser aprovechada en un período anterior al renacimiento?

La salvación de las piezas literarias se realizó durante ochocientos años de activa y responsable tarea de recopilación por parte de los árabes durante la línea de tiempo que oscila entre 650-1450 en la que fueron rescatados, traducidos y retransmitidos hacia los cuatro puntos cardinales.

Los científicos árabes, al igual que Arquímedes o Herón, nunca se separaron del saber empírico, por el contrario, lo profundizaron. El movimiento científico árabe no sólo interpretó el saber de los antiguos sino desarrolló una nueva ciencia donde la razón sustentaba a la fe y viceversa. Esta fue la base esencial que permitió construir el Renacimiento y la Ilustración, que a su vez posibilitaron la Modernidad.

En este contexto, fue de capital importancia la traducción al árabe de la obra de Dioscórides ya que no sólo fue de gran utilidad para la farmacología y medicina en el mundo musulmán sino que a través de éste las recetas del médico griego de Nerón reingresaron a la Europa latina donde fueron recibidas como una panacea. 
El griego Dioscórides, que vivió en el siglo I d.C., está considerado el primer botánico médico científico. Su Materia médica es la mayor obra sobre hierbas y remedios que estuvo en vigencia hasta el siglo XVII. Dioscórides, que fue uno de los grandes pioneros de la medicina natural y la homeopatía, fue rescatado del olvido por los médicos árabo-musulmanes en el siglo X y así pudo estar en vigencia hasta el siglo XVII. Dioscórides, que fue uno de los grandes pioneros de la medicina natural y la homeopatía, fue rescatado del olvido por los médicos árabo-musulmanes en el siglo $\mathrm{X}$ y así pudo estar en vigencia hasta el siglo XVII, curando con sus recetas y consejos a miles de seres humanos de Oriente y Occidente. La obra de Dioscórides sólo entra en la Europa medieval a través de las traducciones de los manuscritos árabes.

Hablar de la herbolaria es hacerlo de la tradición médica que data de 25.000 años atrás, cuando el hechicero o adivino de cualquier tribu conocía remedios para curar algunos males a través de plantas y hierbas. Seguramente en este momento la sensibilidad y el sentido común eran sus aliados en el combate contra la enfermedad. Esta tradición se encuentra en todas las culturas de los cinco continentes del mundo. -

No solamente los chinos y los egipcios recurrieron a la herbolaria para buscar los remedios a múltiples males. En la América Precolombina existía una historia herbolaria que podría tener entre 4.000 y 5.000 años de antigüedad. Incas, aztecas y mayas tenían como base de las curaciones la medicina de hierbas.

La etnobotánica es la disciplina que estudia las relaciones plantahombre, se ocupa de recopilar todos los conocimientos populares sobre plantas y sus usos tradicionales para, posteriormente, interpretar el significado cultural de tales relaciones. Aunque la metodología sigue, en parte, la etnográfica, una correcta interpretación de los datos no es posible sin un sólido conocimiento botánico. Se ha dicho que la etnobotánica es la trama vegetal de la historia de la humanidad. -

Se puede decir también que los primeros estudios etnobotánicos tuvieron lugar en la Antigua Grecia. Allí no sólo surgieron los precursores de la botánica científica sino también de la farmacología. .

La mitología griega marca la aparición de Quirón, un centauro que recibió el conocimiento médico de Apolo; y el cual tuvo varios discípulos, entre ellos, el conocido Esculapio quien con tanto saber, logró obtener la sangre de las medusas y con ésta lograr que los muertos resucitaran. Zeus y Hades se enteraron de este hecho y decidieron sacrificarlo para mantener el equilibrio entre la vida y la muerte, sin embargo Esculapio antes de morir tuvo dos hijas: Higyia, diosa de la medicina y de cuyo nombre se deriva la palabra 
higiene; y Panacea, quien simboliza las propiedades curativas de las plantas, como un remedio para todos los males.

Precisamente, el cuenco o tazón de Higya junto con la serpiente es el símbolo de la farmacia. La palabra farmacología deriva del griego fármakon que significa droga o veneno y logos como discurso o tratado. De farmakía deriva farmacia, farmacéutico, etc.

Teofrastro (372-286), el gran orador, discípulo de Aristóteles -y al que dejo éste su biblioteca y su jardín botánico-, fue el primer gran botánico. Nació en Eresos (una aldea en la isla de Lesbos) y publicó "La historia de las plantas" en nueve libros y "Las causas de las plantas" en seis. En estas obras está contenida la descripción de quinientas especies diferentes y fueron leídas durante casi veinte siglos. En el aspecto médico, dedicó gran atención al estudio de la epilepsia, la peste y al valor terapéutico de muchas plantas medicinales del mundo conocido incluso las de India, Egipto y Libia, posiblemente descubiertas durante las campañas de Alejandro el Grande. Teofrasto significa en griego "divino orador".

\section{El polifármaco por excelencia}

El polifármaco por excelencia en la Antigüedad clásica era la triaca, o teriaca. El término viene del latín Tirus, probablemente derivado del griego Therion, con el significado de serpiente ponzoñosa (de allí el símbolo de la farmacia).

Tal como se utilizaba en el mundo clásico, contenía un número variable de ingredientes, a veces más de setenta. Se realizaron esfuerzos para simplificar la fórmula, pero en el Renacimiento el número de componentes aún se había incrementado. Ideada originalmente como antídoto para la mordedura de animales salvajes, la triaca acabó convirtiéndose en un antídoto universal para los venenos y un remedio habitual para numerosas enfermedades. Entre sus ingredientes, mayoritariamente vegetales, desempeñaba un papel prominente el opio, aunque en diversas épocas la fórmula también incluyó el castóreo, la carne de víbora y la escila.

La más famosa de las triacas era la denominada mithridatium, en honor de Mitrídates VI, rey del Ponto durante la segunda centuria. Para experimentar con sus venenos y antídotos, Mitrídates utilizaba a delincuentes convictos.

El mitrídato que recibió ese nombre por el Rey del Ponto, fue uno de los más famosos contravenenos utilizado en la antigüedad. Incluso el término mitridatismo significa "inmunidad frente a un tóxico". 
Mitrídates VI Eupator el Grande (120-63), rey del Ponto), ensayaba sus fórmulas de venenos, tanto en condenados a muerte como sobre él mismo. Hizo así notables descubrimientos y se convirtió en un experto en el dominio de substancias venenosas, tanto que no pudo quitarse la vida con ningún veneno cuando llegó el momento de su derrota, al ser destruido su palacio en Crimea por Pompeyo el año 63 a.C. tuvo que recurrir a uno de sus esclavos para que le diese muerte con su espada. Al expoliar Pompeyo la Biblioteca del palacio, encontró el gran secreto de Mitrídates, la fórmula de la triaca que era una mezcla de venenos entre los que figuraba el arsénico. Había descubierto y perfeccionado el procedimiento de la inmunización artificial es decir de la seroterapia. Plinio el Viejo lo confirma cuando declara que "fue el primero en mezclar a los antídotos la sangre de los patos del Ponto porque viven de animales venenosos". Cada mañana desde su juventud, asesorado por Crateuas, un experto en venenos que era su consejero, tomaba pequeñas cantidades de diversos venenos para inmunizarse y que no pudiesen envenenarle sus enemigos (mitridatismo).

Uno de los primeros médicos farmacéuticos de la historia fue Nicandro de Colofón. También poeta y gramático, Nicandro de Colofón vivió en el siglo II a.C., en la corte de Atala III, rey de Pérgamo. Su Theriaka o triaca versa sobre las mordeduras de los animales salvajes, serpientes e insectos venenosos, y su Alexipharmaka, sobre otros venenos de origen vegetal y mineral, así como las precauciones que hay que tomar y los remedios propios para su curación. Sus fórmulas mágicas que comprenden de 50 a 60 sustancias fueron aumentadas por Mitrídates, sobre todo con opio y hierbas aromáticas, por Critón, el médico del emperador Trajano, y muy especialmente por Andrómaco, el médico de Nerón.

Andrómaco de Creta fue un médico y cirujano famoso que vivió en el siglo I d.C. Al servicio del emperador Nerón, fue un continuador en la elaboración de la triaca. La triaca de Andrómaco tenía 64 sustancias distintas, incluyendo fragmentos de carne de víboras venenosas, y su preparación era tan complicada que en Venecia en el siglo XV se debía hacer en presencia de los priores y consejeros de los médicos y los farmacéuticos.

Entre sus componentes la teriaca tenía opio, lo que quizá explica su popularidad; la preparación tardaba meses en madurar y se usaba en forma líquida y como ungüento. Otras sustancias que también se recomen daban por sus poderes mágicos eran cuernos de unicornio, sangre de dragón, esperma de rana, bilis de serpientes, polvo de momia humana, heces de distintos animales, etcétera. Andrómaco escribió la fórmula en verso para que no pudiese alterarse fácilmente. 
La paradoja de Nerón era que tenía a Andrómaco para que le preparara una triaca con la que confiaba ponerse a cubierto de cuantos tóxicos pudieran propinarle, y de otra parte, tenía a Locusta, que le proporcionaba ponzoñas para librarse de todas aquellas personas cuyas vidas le representaban un estorbo.

\section{Dioscórides de Anazarbo}

Dioscórides Pedáneo o Pedacio nació en Anazarbo ${ }^{1}$, cerca de Tarso, Cilicia (Turquía), tal vez hacia el año 40 d.C. (Se lo conoce en árabe como Diyusquridis al-Ayn Zarbi). Los escasos datos que sobre él poseemos provienen de la carta que precede a su tratado como prefacio y también como dedicatoria a su amigo Ario, médico de Tarso.

Las menciones a sus contemporáneos y el hecho de que Galeno (c. 129-c. 199) use su obra, permiten deducir que vivió y produjo su obra bajo el mando de Nerón (entre los años 54 y 68). Aunque griego, como la mayoría de los médicos de la época, fue cirujano de los ejércitos romanos en tiempos de Claudio, Nerón y Vespasiano. Estas circunstancias le dieron la oportunidad de viajar y conocer muchas provincias del Imperio Romano (Italia, Galia, Hispania y África del Norte) y de reunir sus propias observaciones sobre los conocimientos que había recibido de sus antecesores.

En la mencionada carta dice: «desde mi temprana juventud, como bien lo puedo afirmar, fui inclinado con un apasionado deseo al conocimiento de la materia médica». Fue contemporáneo de Plinio el Viejo (c. 23-79) y pudo haber muerto hacia el año 90.

\footnotetext{
Anazarbo es una antigua ciudad ciliciana, situada al sudeste de Anatolia, en la moderna Turquía, sobre la llanura Aleian, unos 15 kilómetros al oeste del curso principal del río Pyramus (Ÿihun) y cerca de su tributario Sempas Su. Está a $28 \mathrm{~km}$ al sur del distrito de Kozan, en la provincia turca de Adana. La pequeña aldea que se encuentra en la vecindad fuera de los muros de la antigua ciudad es Dilekkaya. Bajo el Imperio Romano el enclave fue conocido como Cesárea de Anazarbo, a partir de la visita del emperador Octavio Augusto en el año 19 a.C. y fue la principal metrópolis de Cilicia Secunda. Reconstruida por Justino I después de un terremoto, fue llamada Justinopolis (525). Los musulmanes la llamaron Ayn Zarba, y la embellecieron durante la época del califa abbasí Harún ar-Rashíd. El hamdaní Saif ad-Daula (916-967) la fortificó a un gran costo. Sin embargo, fue saqueada por los cruzados. Pero su antiguo nombre persistió y cuando Thoros I, rey de la Pequeña Armenia (1102-1129), derrotó allí a los bizantinos en 1108, la hizo su capital en el siglo XII y la volvió a llamar Anazarbo.
} 


\section{La Materia médica}

Su obra escrita en griego Peri hyles iatrikes, llamada en latín De materia medica, "Materia médica" en castellano, es quizás la obra de medicina más veces reeditada y traducida de la historia. Todavía hoy sigue siendo objeto de interés por numerosos estudiosos. Constituye una fuente indispensable para el estudio de la materia médica, de la botánica, de las creencias populares y también para el estudio de la expresión formal de la prosa científica y del léxico.

La "Materia Médica" no sólo es importante para la historia de la medicina de hierbas sino que además nos permite acercarnos al conocimiento real de las hierbas y remedios utilizados por los griegos, romanos y otras culturas antiguas. El trabajo además identifica los nombres tracios y dacios de algunas plantas que de otra forma se hubiesen perdido. .

En su extenso trabajo que suma cinco libros (cuyo original se perdió en el tiempo), Dioscórides recogió y plasmó el conocimiento acerca de 600 plantas, 90 minerales y 30 productos procedentes de animales, de cada una de las cuales reprodujo un dibujo, indicando sus virtudes terapéuticas o peligrosas. Contiene además 1.000 remedios y 5.000 aplicaciones distintas.

Va recordando en términos simples cual era el uso corriente de las hierbas medicinales siendo una abreviatura de la locución latina medicamentum simples que era el caldo de la técnica griega. Este término, como explicaba Galeno, estaba indicado id quod secundum naturam sincerum est o sea, un medicamento al estilo natural o puro en oposición a medicamentum compositum, o sea, un preparado resultante de la unión de diversas sustancias (compuestos).

Antes de afirmarse la hiatroquímica, entre el siglo XVI y XVII, (que traerá una sustancia no vegetal, sobre todo más de los minerales y metales ), la medicina empleaba como fármacos, especies para suministrar por boca, toda una serie de sustancias derivadas de las raíces, de los bulbos, de las cortezas, de las resinas, de las ye-mas, de las hojas, de las flores, de la floración de los frutos, de las semillas de una cantidad, por nosotros imprevisible de árboles, arbustos, plantas, hierbas y vegetales en general, de una cuidadosa y precisa recogida de los "simples" derivaba por tanto a un buen éxito terapéutico de la actividad de los médicos y farmacéuticos. O sea, usa la importancia de recoger en un herbario, que mostrase en figura y explicase en leyenda, características morfológicas y su correspondiente propiedad terapéutica y orgánica de los vegetales para recoger. 
De aquí la grandeza de la empresa de Dioscórides que entiende el reunir y sistematizar cuanto la práctica empírica y medicina popular sabía alrededor de las plantas y su propio empleo. Con tal fin, consiguió un herbario enciclopédico (con notas también de alimentos, bálsamos y productos cosméticos sobre varios animales, especies venenosas y sus relativos entornos) que junto a los dibujos en color de cada hierba, raíz, plantas medicinales, hasta entonces conocidas (cerca de 600) reunió en capítulos que eran útiles de conocer, o sea, con uno y más nombres con los que venían nombrados, sus propias características y propiedades y su empleo con sus virtudes curativas.

Por extensión y competencia de tradición, tanto botánica como médica, fue el ejemplo y el modelo de los herbarios y de los antídotos farmacéuticos tanto en oriente como en occidente. Su fama se ha conservado intacta desde la era medieval hasta el Renacimiento, con diversas redacciones y muchísimas traducciones.

Entre los grandes y múltiples descubrimientos de Dioscórides figura la utilización de la mandrágora para inducir a la anestesia —en el sentido de ausencia de sensibilidad - en personas que deben recibir un tratamiento quirúrgico o para la cauterización de sus heridas. Aunque esta condición no se refiere a la anestesia general en el sentido moderno, el uso de Dioscórides de la palabra anestesia se anticipa a la utilización del éter por Oliver Wendell Holmes en casi 1800 años ${ }^{2}$.

\section{El Islam y la botánica}

2 Oliver Wendell Holmes (1809-1894) fue un médico estadounidense, especializado en anatomía y fisiología en la Escuela Médica de Harvard donde llegó a ser decano de la institución. El 21 de noviembre de 1846, cuatro semanas después de haber realizado una primera demostración de anestesia con éter en el Hospital General de Massachussets, le escribió una carta a William T. G. Morton (1819-1868), el dentista que había llevado a cabo la operación, donde explica el uso del éter por inhalación. Holmes hizo derivar esa palabra del término griego anaisthesis, que significa insensibilidad o pérdida de sensación con o sin pérdida de conocimiento. Todo había comenzado en 1842 cuando otro estadounidense, el Dr. Crawford Williamson Long extrajo a un paciente un tumor en el cuello después de entumecerle con éter hasta el punto de no sentir nada. Por primera vez en la historia del hombre pudo decirse: se acabó el dolor. Hasta ese día de 1842, el dentista sujetaba bien al paciente en la silla y tiraba de la muela entre los rugidos y la agitación impotente de su víctima, que solía acabar por desmayarse. Long no inventó el éter, descubierto en el siglo XVI por el botánico alemán Valerius Cordus (1515-1544), 300 años antes de que se aprovecharan sus propiedades anestésicas 
Desde el surgimiento de Bagdad a fines del siglo VIII como centro cultural y civilizacional del Islam, todas las ciencias fueron estudiadas y desarrolladas por numerosos eruditos. La botánica no fue una excepción y tuvo además un trato preferencial. -

Los árabo-musulmanes separaron las artes médicas y boticarias, estableciendo en Bagdad a fines del siglo VIII las primeras boticas. Cuando los musulmanes llegaron a España, el sur de Francia y Sicilia llevaron consigo su tradición farmacéutica que fue prontamente asimilada por los europeos.

Es importante citar el Kitab al-nabat (Libro de botánica), de Abu Hanifa Ahmad Ibn Daud al-Dinawari (815-902), de origen persa. Se trata de la obra más completa sobre botánica y agricultura de los primeros tiempos del mundo islámico y que servirá de base a otros textos del mismo género. Está dividida en dos libros: en el primero se describen las plantas que sirven de alimento, plantas de olor, etc.; en el segundo se ofrecen los vegetales en orden alifático, es decir, alfabético.

\section{La Materia médica traducida al árabe}

La botánica y la farmacología musulmanas deben parte de su gran desarrollo a los conocimientos del mé-dico griego Dioscórides. Su "Materia médica" fue traducida al árabe en Bagdad en el siglo IX durante el califato abbasí. Esa traducción, debida a Esteban, hijo de Basilio (861), durante el reinado del califa al-Mutawwkkil, no fue para nada satisfactoria. Fue entonces corregida por el gran traductor Hunayn Ibn Ishãq (808-873), director de la Bait al-Híkma (Casa de la Sabiduría) que la arregló y la hizo manejable. Las palabras griegas que Esteban conocía en árabe las tradujo, pero aquellas que no sabía las transcribió en su forma griega, dejando en manos de Dios el que más tarde hiciera que encontraran alguien que las supiera y pudiera traducirlas al árabe, ya que los nombres de los medicamentos se deben a una convención de las gentes de un mismo país, que son quienes los conocen y les dan nombre, bien por derivación, bien por un acuerdo tácito. Esteban dejó la sinonimia para quienes conocieran las drogas que él desconocía, pues así recibirían los nombres que les convinieran desde el instante en que fueran reconocidas.

Será en el siglo X cuando se lleve a cabo esa traducción óptima. Todo comenzó cuando el emperador bizantino Constantino VII Porfirogénitos ${ }^{3}$,

\footnotetext{
${ }^{3}$ Constantino VII Porfirogénitos, 'nacido en la púrpura') vivió entre 905 y 959. Fue el hijo del emperador bizantino León VI el Sabio. Obtuvo su sobrenombre como hijo legítimo (o más precisamente legitimado) de León, frente a otros que pretendieron el trono durante su vida.
} 
envió una embajada a la corte del califa Abd ar-Rahmãn III de Córdoba (891961) en 949. Entre los presentes dedicados al soberano omeya había un ejemplar de la Materia médica escrito en griego. Éste fue traducido por el monje bizantino Nicolás, enviado también por el propio emperador, al que ayudaron varios médicos andalusíes ${ }^{4}$.

Ascendió a la dignidad imperial a la edad de siete años en 913, bajo la regencia del patriarca Nicolás el Místico. Nicolás se vio obligado a firmar la paz con Simeón de los búlgaros, al que tuvo que reconocer como emperador búlgaro. A causo de ello, Nicolás sería desplazado de la regencia por la madre de Constantino Zoe Karvounopsina. Zoe tampoco consiguió éxito alguno con los búlgaros, y en 919 fue reemplazada por Romano Lecapeno, que casó a su hija Elena con Constantino. Romano se hizo con el poder en solitario hasta 944, cuando fue depuesto por sus hijos, que reconocerían finalmente a Constantino como emperador. En 949 Constantino dispuso una nueva expedición para invadir el Emirato de la isla de Creta, en poder de los musulmanes (provenientes de Córdoba) desde 827, pero al igual que el intento de su padre por reconquistar la isla en 911, fracasó en sus aspiraciones (Nicéforo Focas recuperaría Creta en 961). Este hecho dio lugar a un ataque abbasí por tierra sobre las posesiones bizantinas en Siria, Armenia e Italia, pero los territorios perdidos en el este, serían luego recuperados por Juan Tzimisces, emperador bizantino entre 969-976. Constantino murió en 959 y fue sucedido por su hijo Romano II. Aunque en conjunto la valoración de su reinado es positiva, Constantino resulta más conocido por su faceta de escritor y erudito. Sus buenas relaciones con el califato de Córdoba se debían principalmente a la tradicional amistad omeya-bizantina de dos siglos frente al enemigo común, el califato abbasí de Bagdad.

4 Cuando se habla de España y el Islam, se suele hacer referencia a un concepto con claro significado religioso y a otro con contenido muy directo, de carácter lingüístico. Se habla así, de España musulmana o de España árabe. Sin embargo, en términos populares, con significado antropológico físico en primer lugar, se habla de la España mora. La palabra castellana moro viene, sin duda, del latín «maurus», y del griego «mávros», que significa «oscuro», «negro». Escritores latinos como Juvenal (60-140) y Lucano (39-65) mencionan a los mauros, también conocidos como númidas, que constituían en tiempos de Iugurta (160104), un pueblo caracterizado por su energía física y belicosidad. Recordemos a la famosa caballería númida empleada por los cartagineses en las Guerras Púnicas. La designación étnica en suma, es muy antigua y al principio no tuvo el carácter peyorativo que adquirió después. Parece que la palabra "morisco" se forma como «berberisco», y fue un diminutivo — generalmente peyorativo y descalificador_- que más tarde se empleó para identificar a los hispanomusulmanes que permanecieron en la Península luego de la caída de Granada. Otros sinónimos son moruno, morería, almoraima, morocho, etc. La acepción de beréber, que es otra forma de llamar a los moros, está relacionada con la denominación utilizada por griegos y romanos para designar a los pueblos extranjeros: bárbaros. En la antigüedad clásica el norte de África era conocido como Berbería o país de los bereberes. El país de los mauros o mauritanos se conocía como Mauritania, que luego fue provincia romana y hoy se autodenomina una "república islámica" (aunque en la práctica diste mucho de serlo). Los musulmanes de los siglos VII, VIII y IX aplicaron el nombre de Al-Ándalus a todas aquellas tierras que habían formado parte del reino visigodo: la Península Ibérica, la Septimania francesa y las Islas Baleares. En un sentido más estricto, Al-Ándalus comprenderá la parte de 
El libro atrajo considerablemente la atención del califa y de algunos de sus funcionarios y por esa razón se solicitó al emperador bizantino la ayuda de un traductor idóneo y que además pudiese explicar el contenido de la obra. En 951 el emperador envió a un monje llamado Nicolás quien hablaba correctamente el árabe y que además de ayudar a traducir la obra de Dioscórides y otras obras griegas comenzó a enseñar el griego en la capital a orillas del Guadalquivir. Sus lecturas y explicaciones desencadenaron un gran entusiasmo particularmente entre Hasdai Ibn Shaprut, el canciller del califa y el médico Ibn Ÿulÿul.

\section{Hasdai Ibn Shaprut}

aquellos territorios administrados por el Islam. Conforme avanzaba la conquista cristiana, su extensión se iba reduciendo progresivamente y a partir del siglo XIII designó exclusivamente al reino nazarí de Granada. La prolongada resistencia musulmana granadina contra las incursiones castellano-aragonesas permitirá que se fije el nombre de Al-Ándalus y se perpetúe en el actual de Andalucía. El arabista e islamólogo holandés Reinhart Pieter Anne Dozy (1820-1883) impulsó la teoría que fue apoyada por muchos historiadores modernos según la cual el nombre de Al-Ándalus está relacionado con los Vándalos, suponiendo sin muchos fundamentos, que la Bética pudo llamarse en alguna ocasión Vandalicia o Vandalucía. (Harold Livermore, profesor de Estudios Españoles y Portugueses en la Universidad de Columbia Británica (Canadá) desarrolla el tema en su artículo "La isla de los vándalos", en Actas del segundo Congreso de Hispanistas, Nimega, 1967). Otros argumentan con el nombre germano de "vandalenhaus" (casa o campamento de vándalos) que muy difícilmente pudo haber llegado a oídos de los árabes ya que ese término no se conoció ni siquiera en España. En cambio, la opinión del filólogo español Joaquín Vallvé Bermejo (Univ. Complutense) dice que la expresión árabe Ÿazirat Al-Ándalus (isla de Al-Ándalus) es una traducción pura y simple de "isla del Atlántico" o "Atlántida". (En el uso de los árabes se llama también $\ddot{y} a z i r a h$ (isla, la ÿ suena parecida a la "ye" rioplatense o la "j" inglesa como en John) a las penínsulas e incluso a territorios mesopotámicos). Sin embargo este razonamiento, aunque parece insustancial tiene más lógica que el anterior. (Véase J. Vallvé, La división territorial de la España musulmana, Madrid: Consejo Superior de Investigaciones Científicas, Instituto de Filología, Departamento de Estudios Arabes, 1986; Cap. I: El nombre de Al-Andalus, pp. 17-62). Los textos musulmanes que dan las primeras noticias de la isla de Al-Ándalus y del mar de Al-Ándalus, se clarifican extraordinariamente si sustituimos dichas expresiones por isla de los Atlantes o Atlántida y por mar Atlántico. Lo mismo podemos decir del tema de Hércules y las Amazonas, cuya isla, según los comentaristas musulmanes de estas leyendas grecolatinas, estaba situada en el $\ddot{Y} a u f ~ A l$ Ándalus, lo cual cabe interpretar como al norte o en el interior del Atlántico. La Hesperia (Espéria en italiano) era el nombre con el que se conocían las dos penínsulas más occidentales de Europa: la itálica y la ibérica. Es también en esta latitud extremo-occidental, — según el historiador griego Diodoro Sículo (c. 90-20 aC.) - donde parecen situarse las Hespérides o Atlántidas, los famosos jardines donde se guardaban las manzanas de oro con poderes mágicos. En 716, ya había moneda árabe acuñada en la Península con el nombre de Al-Ándalus. 
El polímata Hasdai Ibn Shaprut (915-970) fue el médico de la corte de Abd ar-Rahmãn III y al-Hãkam II. Esta eminente personalidad judía alcanzó una posición de relevancia política que ningún otro judío había logrado hasta entonces en la España árabe y es el primer personaje hispanohebreo cuya vida y obra conocemos con de-talle. Pertenecía a una importante familia judía oriunda de Jaén y fue su padre Itzhak Ben Ezrá Ben Shaprut, un hombre al parecer muy rico y piadoso, quien decidió trasladarse a Córdoba, la capital de al-Ándalus, y establecerse allí.

Recibió Hasdai una esmerada educación judía y musulmana y puso además su empeño en estudiar medicina, sobresaliendo notablemente en esta ciencia. También mostró un gran interés por los estudios lingüísticos y asimismo dedicó su tiempo al aprendizaje de las lenguas árabe y latina.

Aunque parece que su principal cargo fue ser médico del califa cordobés, desempeñó también otras importantes funciones. Su conocimiento de las lenguas le permitió realizar en ocasiones misiones diplomáticas de éxito para la corte de Córdoba, de modo que también se dejó sentir su influencia en la política exterior del califato.

En el terreno de la medicina colaboró con el grupo que tradujo al árabe el original griego de la "Materia médica" de Dioscórides. Hasdai colaboró estrechamente con el monje Nicolás, pues debido a sus conocimientos de la lengua latina y de la ciencia médica su intervención fue de gran importancia para que la mencionada versión árabe se llevara a cabo.

Respecto al talento diplomático de Hasdai y sus logros en este campo destacaremos su intervención en las negociaciones con el embajador de Otón I Johannes de Gorze que llegó a la capital califal en 956, y con la reina Toda de Navarra, que acudió a Córdoba en el año 958 con su nieto Sancho I el Craso, rey de León (956-958/960-966), que buscaba el apoyo de Abd ar-Rahmãn para recuperar su reino y los servicios médicos de Hasdai y de Abulcasis ${ }^{5}$ para

5 Entre los más importantes cirujanos musulmanes de la época clásica se encuentra Abul Qasim Jalaf Ibn al-Abbas al-Zahraui, también conocido como Abulcasis, quien nació en 936 en Medinat al-Zahara, una localidad a siete kilómetros de Córdoba, en lo que hoy es la Comunidad Autónoma de Andalucía, España. Abulcasis era descendiente de los Ansar ('Auxiliares') del Profeta Muhammad en Medina. Vivió en la corte de Abd ar-Rahmán III, primer Califa de Occidente, en la de su hijo al-Hákam II y en la de su nieto Hishãm II, este último un pusilánime que entregó el poder a su chambelán el ambicioso y autócrata alMansur. Abd ar-Rahmán III fundó la escuela de Medicina de Córdoba, de donde salieron muy buenos médicos. Abulcasis es conocido por sus primeras y originales aproximaciones en cirugía, así como por su famosa enciclopedia médica llamada curiosamente at-Tasrif liman ä̈iza an-Talif ('El saber médico, puesto a disposición del que no ha podido reunirlo'), 
tratado que está compuesto por treinta volúmenes que contienen diferentes aspectos de la ciencia médica. La parte más importante de esta serie comprende tres libros de cirugía, en la cual se describen detalladamente tratamientos quirúrgicos basados en operaciones realizadas por él, que incluyen temas como cauterización, rotura de cálculos de la vejiga, procedimientos ginecológicos, cirugía de ojo, oído y garganta, e intervenciones para la eliminación de fetos muertos, amputaciones y disecciones de animales. Además, este volumen ofrece un gran número de esquemas de más de doscientos instrumentos quirúrgicos, muchos de ellos desarrollados por él. Este libro fue traducido y empleado como texto de estudio en las escuelas de medicina hasta el Renacimiento. El médico andalusí fue el primero en emplear hilo de seda para la suturación de heridas y en ofrecer una completa descripción de la sintomatología y características de la hemofilia. Como experto cirujano maxilar estudió la alineación de los dientes y la forma de corregir los defectos de éstos, así como el implante de huesos de animales para restituir piezas perdidas. Abulcasis centró el estudio y práctica de la cirugía en el saber anatómico y achacó la falta de cirujanos en su época al descuido de la enseñanza de esta parte de la medicina. Su obra es considerada como pionera en cuanto a la explicación de las intervenciones quirúrgicas y a la descripción gráfica del instrumental utilizado, de los cuales tres son notables: aquel que servía para examinar internamente el oído; otro para la inspección de la uretra y el que servía para aplicar o quitar cuerpos extraños de la garganta. Fue el primer hombre que llevó a cabo un procedimiento similar a la laparoscopia, por lo que se le considera el padre de la cirugía moderna. Dentro de su prolífico trabajo, Abulcasis logró introducir un tubo en la vagina de una mujer y, reflejando la luz de un espejo, pudo ver el cuello del útero de su paciente. Abulcasis fue el inventor del fórceps y el catéter de plata. Su Tasrif es el primer tratado de medicina de la historia que tiene ilustraciones de 200 instrumentos quirúrgicos. En su cirugía presenta cerca de doscientas figuras que representan distintos instrumentos quirúrgicos indicando su empleo, lo que ayuda a la mejor comprensión de las técnicas operatorias. Los destinados a la odontología suman un número de veintinueve: cánulas, cauterios, rasorios (16), tenazas, fórceps, dos instrumentos con fines ortodóncicos y alambres de oro para asegurar dientes inestables. Aparte de algunas afecciones máxilo-faciales, se ocupa de enfermedades gingivales, odontalgia, tartrectomía, extracciones (aconsejando la mayor demora posible), malposiciones dentarias (llegando a la extracción si fuera necesario como verdadero tratamiento ortodóncico), o movilidad dentaria post-traumática indicando la inmovilización mediante fijación con alambres a las piezas vecinas. Analizada en su conjunto, la obra, básica para las centurias siguientes, le hace merecedor del calificativo de mejor cirujano oral de su época. Abulcasis completó su Tasrif hacia el año 1000 y fue el resultado de 50 años de estudios médicos y experiencia según sus propias palabras. Describe 325 enfermedades y presenta sus sintomatologías y tratamientos. En los capítulos dedicados a la cirugía describe entre otros procedimientos el tratamiento de las fracturas, las amputaciones y la ligadura de las arterias. En lo que se refiere a las fracturas y luxaciones Abulcasis recoge la tradición hipocrático-galénica, aunque realiza algunas aportaciones propias. Es especialmente relevante la descripción que hace de la utilización de férulas acolchadas y numerosos tipos de vendajes. Utiliza vendajes fenestrados en el caso de fracturas complicadas. También utiliza el yeso en el tratamiento de las fracturas. Abulcasis describe un método novedoso para el tratamiento de las fracturas del pubis en mujeres, intentando evitar la deformidad secundaria que podría originar lesiones durante el parto. Para su reducción introduce en la vagina de la paciente una vejiga de cordero que posteriormente hinchaba hasta conseguir una alineación adecuada de los fragmentos. Asimismo, sus conocimientos como dietista son destacados por los expertos. En el capítulo dedicado a la 
curarse de su hidropesía ${ }^{6}$. La actuación de Hasdai en este caso, como médico y diplomático, dio lugar a una beneficiosa alianza cuya realización se atribuyó a su habilidad y talento.

dietética medicinal, detalla la importancia de cada alimento en relación con su diferente naturaleza. Como farmacéutico, describió abundantes utensilios, desde instrumentos para la elaboración de píldoras o pastillas, hasta los sistemas de filtrado, pasando por la recomendación de las vasijas más adecuadas para una prolongada conservación. El Tasrif, compendió los conocimientos médicos de una época y logró sobrevivir durante siglos. Fue traducido a casi todas las lenguas cultas, entre ellas el hebreo e incluso el provenzal. La traducción al latín por Gerardo de Cremona (1114-1187) tuvo por lo menos diez ediciones entre 1497 y 1544. La última edición es la de John Channing en Oxford (I 778) que contiene el original en árabe y su traducción latina en páginas alternadas. Casi todos los autores europeos de textos quirúrgicos de los siglos XII al XVI se refieren a la cirugía de Abulcasis y la copian. Entre ellos podemos citar a Roger de Salerno (m. 1180), Guglielmo Salicefte (1201-1277), Lanfranchi (m. 1315), Henri de Mondeville (1260-1320), Mondino de Bologna (1275-1326), Bruno de Calabria (1352), Guy de Chaulliac (1300-1368), Valescus de Tarento (1382-1417), Nicolás de Florencia (m. 1411), Leonardo da Bertapagatie de Padua (m. 1460). Abulcasis, este casi desconocido andalusí, que atrajo alumnos y pacientes desde los más lejanos confines del orbe, falleció en el año 1013, poco tiempo después que su ciudad natal fuera saqueada y el califato cordobés cayera estrepitosamente atomizado en 26 taifas o pequeños reinos. No cabe duda que este hispanomusulmán tuvo una influencia mayúscula en el campo de la medicina y la cirugía muy profundamente y que los principios establecidos por él fueron reconocidos como auténtica ciencia, especialmente lo que respecta a la cirugía y sobrepasaron a los de Galeno en el plan de estudios del médico europeo. Abulcasis no sólo fue el más cirujano de los tiempos medievales sino también un gran educador y siquiatra entre otras muchas especialidades.

6 Merece atención especial el tratamiento descrito en el Anexo del Tasrif de Abulcasis mencionado para la obesidad mórbida. La historia es curiosa: el rey Sancho I, rey de León (955-958) y (960-965), era hijo de Ramiro II y de Urraca de Navarra. Se casó con Teresa Ansúrez o de Monzón. Era llamado indistintamente 'El Craso' o 'El Gordo' (era tan obeso que no podía montar a caballo y se le transportaba en un carro). Debido a su enfermedad, no sólo fue objeto de burlas sino que perdió su reino y se vio obligado a refugiarse en el reino de Navarra, al amparo de su abuela, la reina Tota o Toda Aznárez. Ésta estaba emparentada con Abd ar-Rahmán II an-Nasir li-Din Allah (891-961) y le pidió ayuda para tratar la obesidad de su nieto. El califa (cuya madre y abuela eran princesas del reino de Pamplona, por eso era pelirrojo y con ojos azules) lo recibió generosamente y con todos los honores en Córdoba, entregándolo al cuidado de sus dos mejores médicos. Uno de ellos era Abulcasis, el otro el facultativo personal del soberano y canciller el judío Ibn Shaprut. Se aplicó a Sancho un tratamiento dietético y de ejercicios muy intensivo y así, prontamente, se convirtió en un valiente caballero que, de nuevo con la ayuda de Abd ar-Rahmán III, y un ejército musulmán, derrotó al usurpador Ordoño IV y reconquistó el reino de León (Véase Joaquín Vallvé, Abderramán III. Califa de España y Occidente 912-961, Barcelona: Ariel, 2003). Empero, lo pactado por Sancho y Toda con los musulmanes no fue cumplido por aquél, que ya claramente hizo caso omiso de aquella contraprestación, y parecida indisciplina asumieron los navarros al no entregar al conde Fernán González, lo que evidentemente levantó la ira del sucesor de Abd ar-Rahmán, el califa al-Hákam II al-Mustansir y provocó enfrentamientos y 


\section{Ibn Ÿulÿul}

Abu Daud Sulaiman Ibn Hasan al-Andalusi, conocido con el laqab (apodo) de Ibn Ÿulÿul ('hijo del orzuelo'), nació en Córdoba en 943 y murió en la misma ciudad hacia 994. Estudió árabe y ciencias religiosas en su temprana juventud y empezó a interesarse profundamente por la medicina a los catorce años, habiéndose convertido a los veinticuatro en uno de los más importantes médicos.

Fue médico del califa Hishãm II. Además, escribió un comentario a los nombres de las medicinas corrientes mencionadas en la Materia médica de Dioscórides; un tratado acerca de las que no menciona este último, incluidas las que se conocían en al-Ándalus; otro sobre antídotos, y otro sobre las equivocaciones de algunos médicos.

Tiene interés especial su Kitab al-Tabaqat at-tibba ual hukama, "El libro de las categorías de los médicos y los sabios", escrito por mandato del propio califa omeya, y que está dividido en nueve categorías siguiendo la historia de la ciencia desde sus supuestos fundadores Hermes y Esculapio hasta su propia época. Dicho tratado constituye uno de los primeros estudios históricos de la ciencia, en el cual se recogen las biografías de médicos andalusíes y aporta datos sobre la medicina social de la época.

Gracias a él sabemos que los médicos y los fármacos eran gratuitos y proporcionados por las farmacias del palacio. También que las causas de mayor mortalidad entre la población eran debidas a enfermedades de vientre y circulatorias, aparte de las causadas por la guerra.

Hace resúmenes biográficos de médicos y sabios preislámicos e islámicos, Hipócrates, Dioscórides, Platón, Aristóteles, Tolomeo, Euclides,

disputas militares. Pero para entonces Sancho I el Craso ya había entrado en una gran alianza con todos los soberanos peninsulares cristianos que tenía por objeto defenderse de las exigencias cordobesas. En esa alianza tomaron parte León, Pamplona, el conde de Castilla que, por cierto, ya se había olvidado de su títere Ordoño IV, y los condes de Barcelona: Borrell y Mirón. "Esta es la primera vez que Cataluña obra de acuerdo con los demás caudillos cristianos», nos dice Ramón Menéndez Pidal. Las expediciones (aceifas) musulmanas se dirigieron, sin embargo, en contra de lo que parecía pensarse más lógico, hacia aquel núcleo que el califa al-Hákam consideraba más poderoso, y así algunas veces fue contra la frontera castellana, donde parece que tomaron San Esteban de Gormaz (963), percance que obligó al conde Fernán González a pedir la paz, y otras contra la frontera navarra, siendo derrotado, en 967, el rey de Navarra y conde Aragón, García Sánchez I, por al-Tuÿibi, comandante musulmán de Zaragoza. Más allá de la ingratitud del rey leonés, sirvió la experiencia para Abulcasis que la expuso en su manual de medicina. 
Galeno, de los importantes médicos siriacófonos, al-Kindi, ar-Razi y sus colegas andalusíes. Obtuvo su información de fuentes arábigas, de Paulo Orosio (siglo V d. C.), San Jerónimo, Isidoro de Sevilla y, oralmente, de sus contemporáneos. Las "Categorías de Médicos", a su vez, sirvieron de fuente básica a los escritores andalusíes y otros orientales como al-Qifti (1172-1248) e Ibn Abi Usaybiah (1194-1270), siendo también importante el hecho de que Ibn Ÿulÿul contribuyera a la traducción de la "Materia médica" del griego al árabe cuando esta obra fue enviada a Abd ar-Rahmãn III por el emperador de Bizancio. Las Tabaqat son sumamente valiosas para ver con claridad la extensión de los conocimientos y la propagación de las ciencias, lo mismo en Oriente que en al-Ándalus.

Según Ibn Ÿulÿul, la medicina en al-Ándalus estuvo al principio en manos de los cristianos, que confiaban grandemente en el "Aforismo" de Hipócrates y a los que faltaba visión, tanto de su profesión como de filosofía y geometría. Sin embargo, la profesión empezó a tener aceptación e importancia ya en el siglo IX, y lo mismo Ibn Ÿulÿul que Sa'id al-Andalusi de Toledo, que escribió el libro Kitab al-Tabaqat al-Umãn (Libro de las categorías de las naciones), hacen una relación notable de todos a los que consideraban como los más destacados médicos hasta su época ${ }^{7}$.

Ibn Ÿulÿul compondrá en el año 982 su obra titulada Tafsir asma aladwiya al-mufrada min kitab Diyusquridis ('Interpretación de los nombres de los medicamentos simples tomados del libro de Dioscórides'), que es un complemento de la obra clásica.

En la página que identifica los nombre griegos de los medicamentos simples, Ibn Ÿulÿul, realiza esta tarea indicando el mayor número de sinónimos posibles conocidos en distintas lenguas, des-de el árabe hasta el latín, pasando también por otras como el persa, el siríaco y el hindi (hindú). Pero sin lugar a dudas el trabajo más importante al respecto de Ibn Ÿulÿul es el Maqãla fi dikr al-adwiya allati lam yadkur-hã Diyusquridis ('Mención de

\footnotetext{
7 El polígrafo Sa'id al-Andalusi nació en Almería en 1029 y murió en Toledo en 1070. Su obra cumbre conocida en castellano como "Libro de las categorías de las naciones", Kitab Tabaqat al-Umãn (Madrid: Akal, 1999) fue traducida directamente del árabe por el islamólogo y arabista español Dr. Felipe Maíllo Salgado de la Universidad de Salamanca. La obra gozó de un gran predicamento desde su composición, por presentar la situación y expansión de la ciencia entre los diferentes pueblos desde la antigüedad hasta los días del autor, ofreciendo a la vez una historia cultural de los pueblos, con referencias a sus gobiernos, posición geográfica, costumbres y religiones.
} 
los medicamentos que no cita Dioscórides') que es una mejora substancial de la obra del botánico griego.

Es interesante citar las palabras de Ibn Ÿulÿul que nos explican pormenorizadamente como se produjo la llegada del traductor de la Materia médica a al-Ándalus: «El emperador bizantino decía en su carta a an-Nasir (el califa Abd ar-Rahmãn): "No puede obtenerse provecho del Dioscórides más que con un traductor avezado en el griego y que conozca las propiedades de esas drogas. Si tienes en tu país a alguien que reúna estas dos condiciones, sacarás, oh rey, la mayor utilidad de este libro"».

Ibn Ÿulÿul sigue: "Entre los cristianos de Córdoba no había nadie capaz de leer el griego, que es el jonio antiguo. En consecuencia, el libro de Dioscórides se quedó en la biblioteca de Abd ar-Rahmãn an-Nasir sin ser traducido al árabe: estaba en al-Ándalus, pero sus habitantes utilizaban la traducción de Esteban procedente de Bagdad. Cuando an-Nasir contestó al bizantino (Porfiroguénetos), le pidió que le enviase a alguien que hablara el griego y el latín, para que enseñara estas lenguas a sus servidores, que así se transformaron en traductores. El emperador bizantino le envió entonces a un monje llamado Nicolás, que llegó a Córdoba en el año 340 (951). Había entonces en esta ciudad una serie de médicos que investigaban indagaban y buscaban con avidez el modo de determinar los nombres de los simples que figuraban en el Dioscórides y de los cuales aún no conocían su equivalencia en árabe. El más interesado y diligente entre todos esos médicos era el judio Hasdai Ibn Shaprut, quien procuraba complacer a Abd ar-Rahmãn an-Nasir. El monje Nicolás pasó a ser para él la persona más íntima y apreciada. Así pudo comentar los nombres de los simples del libro de Dioscórides que aún eran desconocidos. Fue el primero que fabricó en Córdoba la teriaca llamada faruq, determinando las plantas que entran en su composición. En este tiempo vivían en Córdoba otros médicos consagrados a esclarecer los nombres de los simples que figuraban en dicho libro. Entre ellos se encontraban Muhammad, conocido por al-Sachchar (el Herbolario); otro llamado alBasbasi y Abu Uzman al-Ÿazzar, apodado el Ibicenco; el médico Muhammad Ibn Sa'id, Abd ar-Rahmãn Ibn Ishaq Ibn al-Haytham y Abu Abd Allah alSiqili (el Siciliano) y conocían las propiedades de las drogas».

Termina Ibn Ÿulÿul: «Todos ellos eran contemporáneos del monje Nicolás y los conocí al mismo tiempo que a éste, en la época del reinado de al-Mustansir (al-Hákam II). Yo era en esa época su amigo. Nicolás murió al principio del gobierno de este califa. Gracias a las investigaciones hechas por este grupo de médicos acerca de los nombres de los simples del libro de Dioscórides, llegaron a conocerse en Córdoba, y en todo al-Ándalus, las 
verdaderas propiedades de las plantas, desapareciendo las dudas que se tenían, Se supo exactamente de sus virtudes y el modo exacto como debía pronunciarse su nombre sin cometer errores, excepción hecha de un pequeño número, tal vez diez, lo cual carece de importancia».

\section{Al-Biruni}

Abu ar-Rayhan Muhammad Ibn Ahmad al-Biruni (973-1050), sabio musulmán persa fue además de botánico y padre de la farmacia medieval, astrónomo, historiador, geólogo, poeta, filósofo, matemático, físico y geógrafo, autor del famoso Kitab fi Tahqiq ma li’l-Hind ('Libro de investigaciones sobre la India').

En uno de sus libros de farmacia, as-Saydanah fit-Tibb ('La farmacia en la medicina'), al-Biruni define as-Saydanani (el farmacéutico) como el profesional especializado en coleccionar drogas seleccionado las mejores de las simples o las compuestas y en la preparación de remedios.

Al-Biruni confirma que la palabra saydanani proviene del sánscrito o jandanani. En India la madera de sándalo (sánscrito, jandan) era usada extensivamente, más que otras maderas olorosas.

En árabe la persona que vende ámbar es llamado anbari, de manera que la persona que comerciaba con el sándalo fue llamado sandanani y más tardíamente sandalani, saydalani o saydali.

De la misma forma las boticas (al-'attar), en árabe fueron llamadas ad-dari, debido a que los barcos que transportaban almizcle, perfumes y especias de la India atracaban en el puerto de Dhahrãn (hoy Arabia Saudí).

Los boticarios árabes ( 'attarin) que vendían perfumes y aromas no utilizaban el sándalo tan frecuentemente como los indios. Ellos excluyeron el sándalo, primariamente, porque no era popular en Arabia. Por esa razón, aplicaron el nombre de sandalani (que de acuerdo al-Biruni es el término más apropiado) a los más calificados farmacéuticos que se especializaban en drogas y medicamentos simples y remedios compuestos. -

La palabra droga ('uqqar), dice al-Biruni, viene del siríaco y es la denominación para un tocón de árbol (parte del tronco que queda unido a la raíz cuando se corta un árbol por el pie), o sea raíz, en griego rizoma). Esta palabra ('uqqar) fue luego aplicada a todas las partes del árbol y fue tomada por los árabes para identificar la materia médica (en plural 'uqaqir).

\section{Ibn al-Wafid}


Abu-l-Mutarrif Abd al-Rahmãn Ibn Muhammad Ibn Abd al-Kabir Ibn Iahya Ibn al-Wafid Ibn Muhammad al-Lajmi (1008-1074), el Abengüefith de los farmacólogos medievales, nació en Toledo. Compuso varias obras de medicina, entre ellas el Kitab al-adwiya al-mufrada ('Libro de los medicamentos simples') basado en Dioscórides, además de un Kitab al-Wisãd ('Libro de recetas').

Se hizo famoso en toda Europa gracias a la traducción resumida de Gerardo de Cremona: Liber Abenguefith Philosophi de virtutibus medicinarum et ciborum. Fue traducido completamente al castellano y al catalán.

Ibn al-Wafid estuvo al servicio de al-Ma'mûn, régulo de Taifa de Toledo (1037-1075) y para él creó un jardín botánico o "Huerta del Soberano" (Ÿannat as-Sultãn), que se extendía por la Vega del Tajo, entre el Palacio de Galiana y el Puente de Alcántara. Fue en este siglo XI, cuando aparecieron en al-Ándalus los primeros "Reales Jardines Botánicos", casi quinientos años antes que en la Europa renacentista.

Entre otras obras, Ibn al-Wafid escribió una "Suma o compendio de Agricultura", cuya versión castellana de la Edad Media descubrió e identificó el arabista-hebraísta catalán José María Millás i Vallicrosa (1897-1970) en el manuscrito fragmentario de la Biblioteca Nacional de Madrid, procedente del antiguo fondo de la Biblioteca de la Catedral de Toledo.

La obra agronómica de Ibn al-Wafid inspiró uno de los más famosos tratados de agricultura del Renacimiento: la "Agricultura General” de Gabriel Alonso de Herrera, editada en 1513 por encargo del Cardenal Francisco Jiménes de Cisneros.

\section{An-Nabati}

Abu al-Abbas Ahmad Ibn Muhammad Ibn Mufarraÿ an-Nabati (11651240) nació y murió en Sevilla. Estudió en Marrakesh con el farmacéutico Ibn Salih y fue autor de varios tratados de botánica. En su Kitab al-Rihla alNabatiyya ('Libro del viaje botánico') narra su periplo por Oriente y, al mismo tiempo, proporciona una descripción de las plantas que va hallando en el camino; su obra se ha perdido, aunque se conservan citas en la de su discípulo Ibn al-Baytar.

\section{Abu-l-Salt de Denia}


Abu-1-Salt de Denia (1067-1134), que además de médico fue astrónomo y matemático y residió en Sevilla, El Cairo y Mahdiyya (Túnez), fue autor de un Kitab al-adwiya al-mufrada traducido al latín por el ilustre médico valenciano Arnau de Vilanova (ca. 1238-1311). Idéntico título llevan las obras de Abu-l-Ala Sur Ibn Abd al-Malik Ibn Muhammad (m. 1130), padre de Ibn Zuhr (1095-1161), (el famoso Avenzoar de los latinos), médico de al-Mutamid, rey de la taifa de Sevilla, y luego ministro del emir almorávide Yusuf Ibn Tashfin, y la de Abu Ÿafar Ahmad Ibn Muhammad alGafiqi (m. 1165), hijo del célebre oculista cordobés Muhammad al-Gafiqi, quien describe muy cuidadosamente las plantas, dando sus nombres en árabe, latín y beréber.

\section{Ibn al-Baytar}

El más grande botánico farmacólogo de la civilización islámica fue Diya al-Din Abu Muhammad Abdallah Ibn Ahmad, llamado Ibn al-Baytar ('hijo del veterinario'), al-Malaqui ('el Málagueño'), discípulo de an-Nabati.

Nació en Benalmádena, en 1197, entre Málaga y Marbella, sobre la costa del Sol, donde vivió su niñez y adolescencia y donde comenzó a interesarse por las plantas de su entorno y la forma de utilizarlas para curar ciertos males. Estudió en Sevilla y en 1220, con 24 años de edad dejó alÁndalus para instalarse en el Oriente musulmán y encontrar la muerte en Damasco en 1248.

En la ciudad de El Cairo, el sultán ayubí Malik al-Kamil Nasiruddín Muhammad, sobrino del famoso Salahuddín (Saladino), que gobernó entre 1218-1238, lo nombró jefe de los herboristas de palacio y fue probablemente allí donde escribió sus obras más importantes.

Ibn al-Baytar viajó luego a Siria y Anatolia, a pesar de las invasiones cruzadas, para recoger hierbas, y sus trabajos constituyen la mejor sistematización sobre las plantas medicinales que jamás se emprendió antes de la época moderna. En Damasco llegó a ser Visir con grandes honores y siendo una de las personas más influyentes de todo el territorio.

Sus estudios de botánica, zoología, mineralogía y de la naturaleza del hombre le hacen publicar un elevado número de obras consideradas como guías para los sabios que le siguieron. En su gran enciclopedia Al-Ÿami limufradat al-adwiya ua-l-agdiya ('Colección de nombres de alimentos y drogas simples') hace una relación de más de 1400 ejemplos de medicinas naturales, hierbas, remedios y alimentos productos de su propia observación trescientas de las cuales nunca se habían inventariado hasta entonces-, así 
como la consulta de más de 150 fuentes y una relación sistemática, por orden alfabético, de sus estudios e investigaciones sobre medicamentos y alimentos contenidos en los tres reinos naturales. También cita a los autores griegos y latinos y anota sus propias observaciones y críticas.

Se trata, en fin, de un repertorio crítico del conjunto de la ciencia farmacológica que permaneció como el fundamento de toda la botánica ulterior en el Oriente musulmán.

En su Tafsir Kitab Diyusquridus ('Comentario de las plantas que aparecen en el libro de Dioscórides'), Ibn al-Baytar analiza la 'Materia médica' del botánico heleno.

\section{Traductores occidentales de Dioscórides}

La obra de Dioscórides estuvo vigente hasta fines del siglo IV d.C. A partir de entonces la mayoría de los manuscritos griegos y latinos fueron destruidos por teólogos fanáticos, principalmente en el saqueo del Serapión y la Biblioteca de Alejandría en 391. Los archivos de Roma fueron devastados en sendas ocasiones entre 410 y 476 por alanos y hérulos, y los escasos vestigios clásicos que quedaban en Atenas fueron arrasados por Justiniano I en 529. Unas pocas obras se salvaron en Bizancio pero quedaron confinadas a la elite de la corte de Constantinopla. Entre ellas figuraba la obra de Dioscórides guardada en un manuscrito fechado en 512 Juliana Anicia Codex (ca. 512 A.D.), que hoy forma parte de la Biblioteca Nacional de Austria en Viena. Registrado como Codex Vindobonensis Medicus Graecus 1, se lo conoce mejor como "Viena Dioscórides", la más antigua y valiosa obra en la historia de la botánica y la farmacología.

La traducción al árabe realizada en conjunto por Nicolás, Ibn Shaprut e Ibn Ÿulÿul no sólo tuvo una distribución fluida y masiva por todo el mundo islámico sino que oportunamente sería reenviada a la Europa cristiana y traducida al latín. Además la versión árabe fue enriquecida por las correcciones y los comentarios de Ibn Ÿulÿul, Ibn al-Wafid e Ibn al-Baytar entre otros. Entre las ediciones latinas podemos contar la de Siena (1478), París (1516) y Salamanca (1566). La primera versión en griego sería la de Aldus Manutius en Venecia (1499), la primera versión castellana se realizó gracias a Andrés Laguna en 1555 y la primera traducción al inglés fue obra de John Goodyer en 1655.

\section{BIBLIOGRAFÍA}


Álvarez Millán, Cristina. "Medical Anecdotes in Ibn Juljul's Biographical Dictionary", en Suhayl (Journal for the History of the Exact and Natural Sciences in Islamic Civilization, 4, 1-18. Barcelona: Universidad de Barcelona, 2004.

Dioscorides. De Materia Medica. Being an Herbal with many other Medicinal Materials written in Greek in the First Century of the Common Era. A New Indexed Version in Modern English by Tess Anne Osbaldeston and Introductory notes by Rob P. Wood. Johannesburg: Ibidis Press, SA, 2000. $932 \mathrm{pp}$.

Dioscorides Pedanius. Dioscorides: Codex vindobonensis medicus graecus 1 of the Austrian National Library. Graz: Akademische Druckerei und Verlagsanstalt, 1977.

Freely, John. Aladdin's Lamp: How Greek Science Came to Europe Through the Islamic World. New York: Knopf, 2009.

Garijo, Ildefonso. "El Tratado de Ibn Yulyul sobre medicamentos que no mencionó Dioscórides", Ciencias de la Naturaleza en al-Andalus. Textos y Estudios. I, editados por Expiración García Sánchez. Granada: CSIC, 1990, pp. 57-70.

Garijo, Ildefonso. Ibn Yulyul. Tratado Sobre los Medicamentos de la Triaca. Córdoba: Servicio de Publicaciones de la Universidad de Córdoba, 1992.

Graham, Mark. How Islam Created the Modern World. Beltsville: Maryland: Amana, 2006.

Gutas, Dimitri. Greek Thought, Arab Culture: The Graeco-Arabic Translation Movement in Baghdad and Early 'Abbasid Society. London and New York: Routledge, 1998

Ibn Wafid. El libro de la almohada, trad de Camilo Álvarez de Morales, Instituto Provincial de Investigaciones y Estudios Toledanos, Toledo, 1980.

Ibn Wafid. Kitab al-adwiyya a-mufrada = Libro de los medicamentos simples, edición, traducción, notas y glosario de Luisa Fernanda Aguirre de Cárcer. Madrid: Consejo Superior de Investigaciones Científicas, Agencia Española de Cooperación Internacional, 1995.

O’Leary, DeLacy. How Greek Science Passed to the Arabs. London: Taylor and Francis, 2001.

Pedacio Dioscórides Anazarbeo. Acerca de la Materia Medicinal, y de los venenos mortíferos, traduzido de lengua griega, en la vulgar castellana (Mathías Gast, Salamanca, 1566) por Andrés de Laguna. Aranjuez: Edición facsímil, 1999. 
Pedanius Dioscorides. Sobre Los Remedios Medicinales: Manuscrito de Salamanca: Estudios y Traducción. Salamanca: Ediciones Universidad Salamanca, 2006.

Riddle, John M. Dioscorides on Pharmacy and Medicine. Austin, TX: University of Texas Press, 1986.

Turner, Howard R. Science in Medieval Islam: An Illustrated Introduction. Austin, TX: University of Texas Press, 1997. 